\title{
Prevalencia del dolor musculo-esquelético según la estrategia COPCORD en una población urbana del cantón Azogues, Ecuador
}

\section{Musculoskeletal pain prevalence according to COPCORD strategy in an urban population from the Azogues canton, Ecuador}

\author{
Beatriz Patricia Quichimbo Miguitama ${ }^{1 *}$, Ana Belén Cárdenas Carangui ${ }^{1}$ \\ ${ }^{1}$ Universidad Católica de Cuenca \\ *bpquichimbom@ucacue.edu.ec
}

DOI: https://doi.org/10.26871/killkana_salud.v4i1.582

\begin{abstract}
Resumen
Introducción: Las enfermedades reumáticas constituyen la principal causa de dolor músculo-esquelético y son responsables de limitación física y discapacidad. El objetivo principal de este estudio es estimar la prevalencia del dolor músculoesquelético en una población urbana del Cantón Azogues mediante la implementación de la etapa I de la estrategia COPCORD. Metodología: Estudio descriptivo, de corte transversal, basado en la aplicación del cuestionario COPCORD core, versión 2-mexicana en adultos mayores de 20 años, del Barrio Zhirincay, de la ciudad de Azogues, para detección de dolor músculo-esquelético secundario a enfermedades reumáticas. Resultados: se aplicó el cuestionario a 197 personas; la prevalencia de dolor músculo-esquelético fue de 61.93\% (IC 95\%: 57.03-66.); es más frecuente en mujeres (59\%; P: 0.184); y en adultos mayores de 66 años (91\%, P: 0.00567). El $41.80 \%$ experimentó dolor de intensidad moderada y el $39.34 \%$ dolor de gran intensidad; el $40.98 \%$ (P: 0.566) ha presentado limitación funcional en algún momento de su vida; el $3.28 \%$ presenta discapacidad. La frecuencia de absentismo laboral a causa del dolor fue del $59 \%$ (P: 0.06). Solo el $45.08 \%$ ha tenido una evaluación y diagnóstico previo de enfermedad reumática y el $47.54 \%$ recibe algún tratamiento. Las zonas más frecuentes de dolor son: región dorso-lumbar 36,7\%, cervical 29\% y rodillas 16,39\%. Conclusión: Mediante el cuestionario COPCORD se detectó una alta prevalencia de dolor músculo-esquelético en esta población, la misma se incrementa con la edad y constituye una causa importante de limitación funcional y discapacidad que requiere una detección temprana y tratamiento oportuno.
\end{abstract}

Palabras clave: enfermedades reumáticas, COPCORD, discapacidad física.

\begin{abstract}
Introduction: Rheumatic diseases constitute the main cause of musculoskeletal pain, and are responsible for physical limitation and disability. This study aims to estimate musculoskeletal pain prevalence in an urban population from the Azogues canton, through the implementation of stage I Community Oriented Program for Control of Rheumatic Diseases (COPCORD) strategy. Methodology: This is a descriptive, cross-sectional study based on the application of COPCORD core, Mexican questionnaire, version-2. The survey was applied to adults over 20 years, from the Zhirincay neighbourhood located in Azogues, to detect musculoskeletal pain secondary to rheumatic diseases. Results: The questionnaire was administered to 197 people; the prevalence of musculoskeletal pain was 61.93\% (CI 95\%: 57.03-66.); being more frequent in women (59\%; P: 0.184); and in adults over 66 years (91\%, P: 0.00567). 41.80\% of the people experienced moderateintensity pain while $39.34 \%$ experienced high-intensity pain; $40.98 \%$ (P:0.566) have presented functional limitation at some point of their lives and $3.28 \%$ present disability. Work absenteeism frequency due to pain was $59 \%(P: 0.06)$. Only $45.08 \%$ have had a previous rheumatic disease medical evaluation and diagnosis, $47.54 \%$ are under treatment. The most frequently painful areas are the dorsal-lumbar 36.7\%, cervical 29\%, and knees 16.39\%. Conclusion: Through the use of the COPCORD questionnaire, a high musculoskeletal pain prevalence was detected in this population. It increases with age and constitutes an essential cause of functional limitation and disability that requires early detection and timely treatment.
\end{abstract}

Keywords: rheumatic diseases, COPCORD, physical disability. 


\section{Introducción}

El dolor músculo-esquelético (DME) se define como aquel que tiene su origen o se percibe en los músculos, los huesos o sus tejidos adyacentes, como fascias, tendones, ligamentos, membranas sinoviales, etc. ${ }^{1,2}$ Es producto de diversas afecciones reumatológicas sean inflamatorias (artritis reumatoide, artritis por depósitos de cristales, lupus eritematoso sistémico, espondiloartropatias, etc.) o no inflamatorias (osteoartritis, osteoporosis, fibromialgia, neoplasias). ${ }^{2,3}$ Se lo debe diferenciar del dolor miofascial que se caracteriza por la contracción muscular dolorosa en banda desencadenado al estimular los puntos gatillos y se acompaña de sintomatología sensitiva, motora y autonómica local o a distancia del punto gatillo, se presenta de manera insidiosa o como respuesta a un trauma o lesión., ${ }^{4,5}$

La prevalencia del DME varía entre el 13.5 a $47 \%$, dependiendo de la población estudiada $;^{2}$ se incrementa con la edad, desde el $5 \%$ en menores de 15 años a mas del $31 \%$ en mayores de 50 años, afectando con mayor frecuencia a las mujeres, ${ }^{3}$ se estima su aumento en las décadas siguientes debido principalmente al incremento de la expectativa de vida y al envejecimiento de la sociedad; 6 las enfermedades reumáticas, como principal causa de DME tiene una prevalencia alta, se ubican entre las enfermedades no transmisibles mas frecuentes y constituyen una causa habitual de ausentismo laboral ${ }^{7}$ y primera causa de discapacidad en los países desarrollados ${ }^{8,9}$ debido a que de manera común afectan el aparato locomotor manifestándose principalmente con dolor, rigidez e inflamación crónica y persistente; en ocasiones se acompaña de compromiso sistémico, multiorgánico secundario a fenómenos autoinmunes; en estos pacientes también se reporta una mayor prevalencia de síntomas de ansiedad y depresión ${ }^{8}$ todo esto conduce al deterioro de la capacidad funcional, al incremento de la mortalidad (pérdida de años de vida) y morbilidad (años vividos con discapacidad) con reducción de la productividad, de los ingresos familiares ${ }^{10}$ y deterioro de la calidad de vida, ${ }^{11}$ generando un impacto socioeconómico importante para el paciente, la familia y la sociedad en general. ${ }^{9,12,13}$

La carga de la enfermedad se incrementa al ser una causa frecuente de consulta en los servicios de atención primaria y urgencias ${ }^{14}$ y requerir frecuentes hospitalizaciones y cuidados domiciliarios especiales, ${ }^{8}$ el costo de las mismas representa entre el 1 y $3 \%$ del producto interno bruto, conformando un problema de salud pública de alto impacto; ${ }^{1,11,15}$ de ahí la importancia de la detección, diagnóstico temprano y el tratamiento rápido y efectivo con el propósito de disminuir o revertir la discapacidad física y social que producen; ${ }^{16}$ con este objetivo en 1981 la Organización Mundial de la Salud y la Liga Internacional de Asociaciones de Reumatología (WHO-ILAR) desarrollaron el Programa Orientado a la Comunidad para el Control de Enfermedades Reumáticas (Community Oriented Program for Control of Rheumatic Diseases COPCORD) como una estrategia epidemiológica, eficaz y de bajo costo diseñada para la identificación, prevención y control de las enfer- medades reumáticas en países en vías de desarrollo, $, 9,11,17$ especialmente para las áreas rurales; ${ }^{18}$ ha sido validado en varios idiomas y culturas en diversos países, adaptándolo a las condiciones locales; ${ }^{16}$ pretende mejorar la educación en salud, la calidad de los servicios médicos y eventualmente reducir la carga de la enfermedad; esta herramienta enfatiza el registro de síntomas (dolor, inflamación y rigidez) y discapacidad mas que enfermedades y síndromes. ${ }^{18}$

La sensibilidad de la estrategia COPCORD para detección de enfermedades reumáticas depende de la prevalencia de la enfermedad en la población de estudio, variando en América desde el 51,7\% hasta el $84 \%$ con especificidad del $35 \%$ al $80 \%,{ }^{12,16}$ su aplicación en poblaciones indígenas de Latinoamérica demostró una sensibilidad del 73,8\% y especificidad del $72.9 \%$ cuando se la compara con la evaluación clínica por un Especialista Reumatólogo aplicando los criterios clínicos para diagnóstico de enfermedades reumáticas de la American College of Rheumatology. La sensibilidad para cada enfermedad reumática varia del 60 al $100 \%$ y la especificidad del 60 a $66 \%$, detectándose por esta metodología: osteoartritis $(80.65 \%$ de sensibilidad y $66 \%$ de especificidad); dolor lumbar $(60.71 \%$ y $61,76 \%$ de sensibilidad y especificidad respectivamente), artritis reumatoide (sensibilidad $90.91 \%$ y especificidad $60,78 \%$ ); artritis no diferenciadas (Sensibilidad $83.33 \%$ y especificidad 60,34\%), espondiloartritis (Sensibilidad 100\% y especificidad $60.14 \%) .{ }^{17}$

La estrategia COPCORD Tiene 3 fases: Fase 1 o epidemiológica: su objetivo es identificar a los pacientes con síntomas osteomusculares de origen no traumático, mediante la aplicación del cuestionario COPCORD Core (CCQ). Fase II corresponde al tratamiento de los casos confirmados mediante una valoración clínica por un Reumatólogo y la implementación de estrategias educativas. Fase III tiene como finalidad detectar los factores de riesgo ambientales y genéticos de las enfermedades reumáticas. ${ }^{10,18}$

En nuestro medio no hay estudios sobre la prevalencia de estas patologías ni de sus consecuencias, por lo que se consideró importante disponer de estadísticas locales; siendo el objetivo principal de este estudio estimar la prevalencia de dolor músculoesquelético en una población urbana del Cantón Azogues mediante la implementación de la fase I de la estrategia COPCORD, una herramienta aceptada internacionalmente y validada en nuestro país.

\section{Materiales y métodos}

Población de estudio. - esta constituida por todos los adultos mayores de 20 años (197 personas), residentes en el Barrio Zhirincay correspondiente a la zona 3 del cantón Azogues de la Provincia del Cañar, el mismo que cuenta con una población total de 412 habitantes (información proporcionada por el Municipio).

\section{Metodología}

Se procedió a la socialización del estudio a la población en general y la aceptación personal fue registrada en un consentimiento informado escrito. 
Se aplicó en el domicilio el cuestionario COPCORD (versión 2-mexicana), el mismo que consta de las siguientes secciones: información demográfica, comorbilidades auto reportadas, historia laboral, malestar o dolor músculoesquelético en los últimos 7 días, dolor en algún momento de su vida, localización del dolor en el maniquí, intensidad del dolor medida por una escala visual análoga del 0 al 10, limitación funcional y absentismo laboral.

Se definieron como casos positivos aquellos que reportaron DME mayor de 1 en la escala visual análoga en los últimos 7 días o dolor en algún momento de su vida, se excluyó el dolor asociado a trauma, también se consideró como positivo si ha presentado rigidez e hinchazón en alguna articulación en los últimos 7 días.

Los resultados del cuestionario fueron informados a la población para su derivación a un centro médico para completar la valoración médica y los estudios pertinentes.

Análisis estadístico: se utilizó medidas de tendencia central y dispersión en variables continuas y frecuencias absolutas y relativas en variables ordinales, nominales o categóricas, para las comparaciones se utilizaron pruebas paramétricas y no paramétricas de acuerdo a las características de los datos.

\section{Resultados}

Características demográficas: La población está compuesta por 197 adultos mayores de 20 años, sus características se exponen en la tabla 1; 109 (55.3\%) son mujeres; el grupo de edad más frecuente se ubica entre los 41 y 50 años con 62 personas $(31.47 \%$ ) y el menos frecuente fue el grupo de más de 66 años con 22 individuos $(11.1 \%)$; 93 personas son casadas $(47.2 \%)$. La mayor parte de la población $(87.3 \%)$ presenta alguna comorbilidad, siendo las enfermedades cardiacas las más prevalentes estando presentes en 56 personas $(28.4 \%)$.

Tabla 1. Características sociodemográficas de la población

\begin{tabular}{|c|c|c|c|}
\hline Variables & & $\mathrm{n}$ & $\%$ \\
\hline Mujer & & 109 & 55.3 \\
\hline Edad & $41-50$ años & 62 & 31.47 \\
\hline \multicolumn{4}{|c|}{ Estado civil } \\
\hline & Casado & 93 & 47.2 \\
\hline & Soltero & 50 & 25.4 \\
\hline & Divorciado & 16 & 8.1 \\
\hline & Viudo & 16 & 8.1 \\
\hline & Unión libre & 22 & 11.2 \\
\hline \multicolumn{4}{|c|}{ Intensidad laboral } \\
\hline & Leve & 63 & 32.0 \\
\hline & Moderada & 91 & 46.2 \\
\hline & Severa & 43 & 21.8 \\
\hline \multicolumn{4}{|c|}{ Lugar de trabajo } \\
\hline & Urbano & 135 & 68.5 \\
\hline & Rural & 62 & 31.5 \\
\hline \multicolumn{4}{|c|}{ Comorbilidades } \\
\hline & Enfermedades cardiacas & 56 & 28.4 \\
\hline & Diabetes mellitus & 42 & 21.32 \\
\hline & Enfermedad renal crónica & 7 & 3.55 \\
\hline & Enfermedad neuromuscular & 29 & 14.72 \\
\hline & Cáncer & 3 & 1.52 \\
\hline & Otros & 35 & 17.8 \\
\hline & Ninguna & 25 & 12.69 \\
\hline \multicolumn{4}{|c|}{ Absentismo laboral } \\
\hline & $\mathrm{Si}$ & 116 & 58.9 \\
\hline
\end{tabular}

En relación a la actividad laboral, 135 (68.5\%) encuestados laboran en el área urbana siendo el $53 \%$ mujeres; la mayoría realiza trabajos de moderada intensidad $46.19 \%$ sin encontrar relación con el género (P: 0.43) ni con el área de trabajo (P: 0.63).

El COPCORD fue positivo para dolor en los últimos 7 días en 49 personas (40.61\%; IC 95\%: 33.75-47.47) y para dolor en los últimos 7 días más dolor histórico en 122 personas (prevalencia del 61.93\%; IC 95\%: 57.03-66.0); sus características se exponen en la tabla 2.

Tabla 2. Características de la población con COPCORD positivo

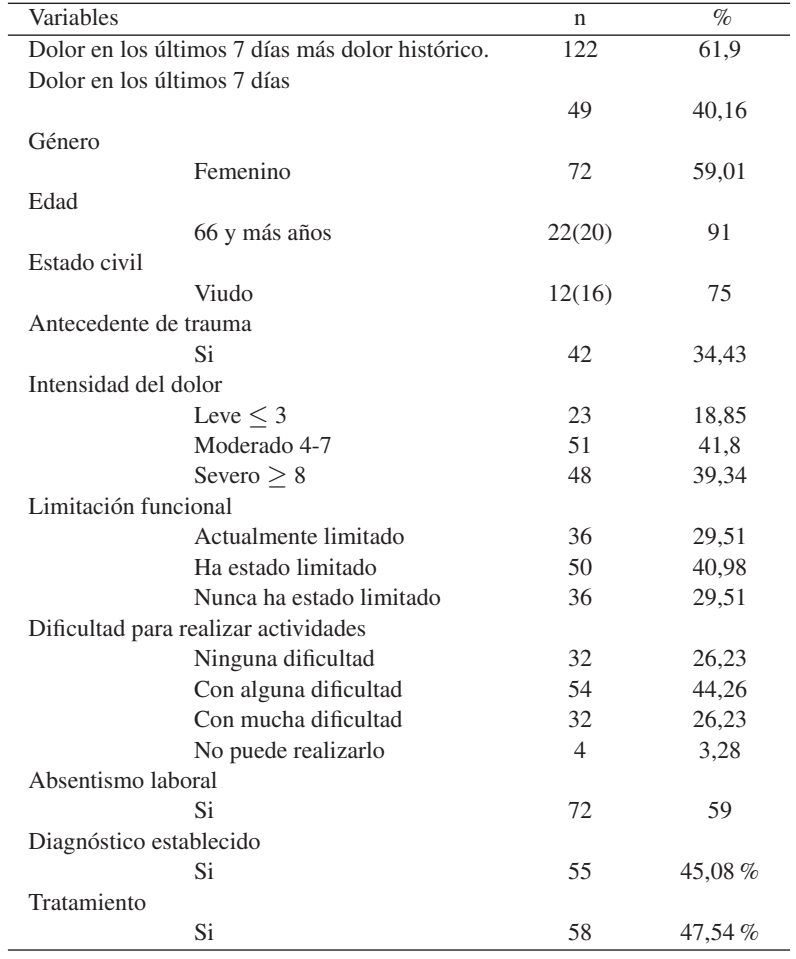

El género más afectado es el femenino, con 72 personas (59\%; P: 0.184); es más frecuente en los grupos de mayor edad, está presente en 20 de 22 personas mayores de 66 años (91\%; P: 0.00567) y es menor en el grupo de 20-29 años (46\%). Figura 1.

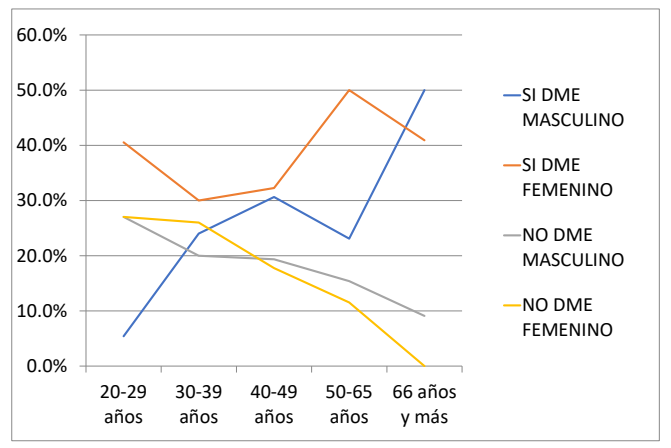

Fig. 1. Distribución del dolor músculo-esquelético de acuerdo al género y la edad 
El DME se evidencia en 12 de 16 (75\%; P: 0.39) individuos viudos y en menor frecuencia en los que viven en unión libre 10 de 22 (45\%).

El COPCORD positivo se exhibe con mayor frecuencia cuando se realizan actividades de intensidad leve $66 \%$ y moderada $62 \%$ (P: 0.383); y es similar en el área urbana y rural $(62 \%$ y $61 \%$ respectivamente, P: 0.90). Figura 2.

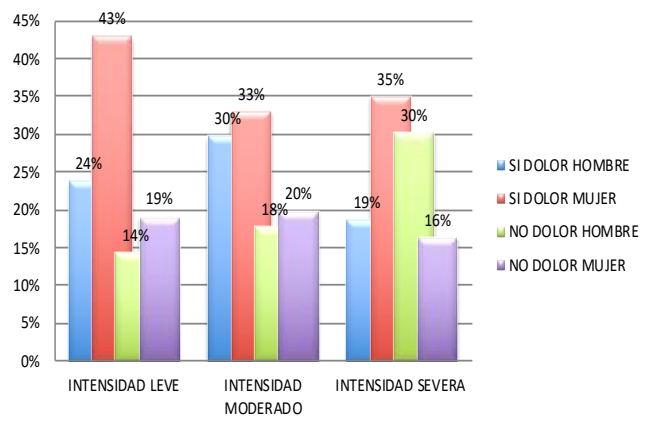

Fig. 2. Frecuencia de dolor músculo-esquelético de acuerdo a la intensidad laboral

El DME está presente en todos los casos con comorbilidades autoreportadas, predominando en quienes padecen cáncer $(100 \%)$. Figura 3.

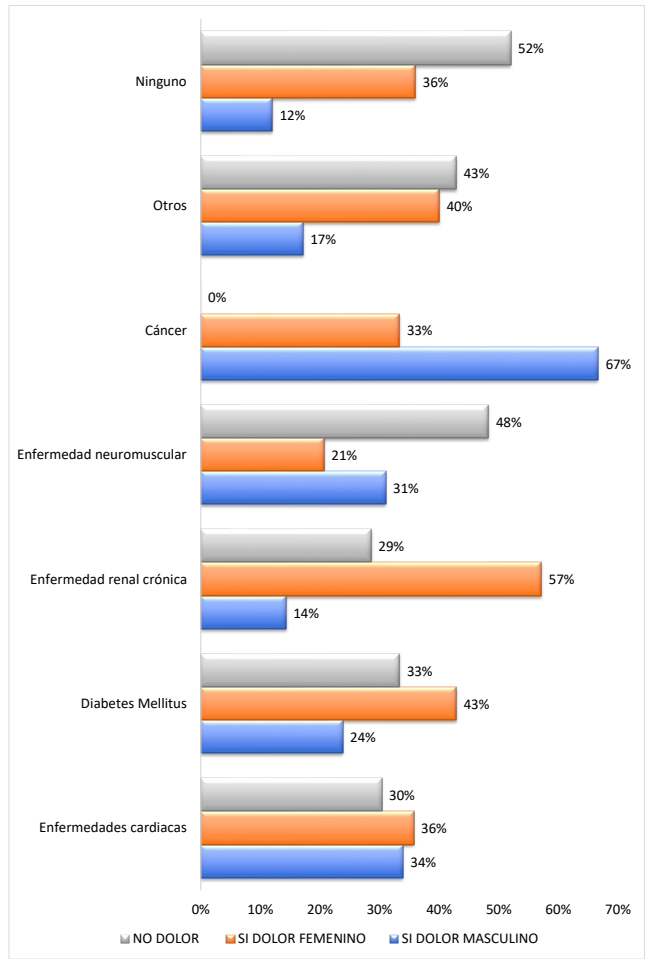

Fig. 3. Dolor músculo-esquelético y comorbilidades autoreportadas

En relación a la intensidad del dolor, 48 casos (39.34\%; P: 0.096) experimentaron dolor severo, catalogado en la escala visual análoga como mayor a 8 puntos; solo 55 casos
$(45.08 \%)$ tienen evaluaciones médicas previas, siendo los diagnósticos mas usuales la osteoporosis y osteoartritis y el $55.3 \%$ tiende a auto medicarse.

De los encuestados con COPCORD positivo, 50 personas (40.9\%; P: 0.566) han presentando en algún momento limitación funcional y $4(3.27 \%)$ tienen discapacidad; la prevalencia de absentismo laboral es del $59 \%$ (IC $95 \%$ : 50.29-67.74; P: 0.961).

El sitio de dolor más frecuente en los varones es la región cervical, presente en 19 personas (38\%) seguido de la región dorso-lumbar, (26\%) y rodillas (20\%); a diferencia de las mujeres en quienes la región dorso-lumbar constituye el principal lugar afectado $(43 \%)$ seguido de la región cervical $(24 \%)$ y rodillas $(14 \%)$. Figura 4.

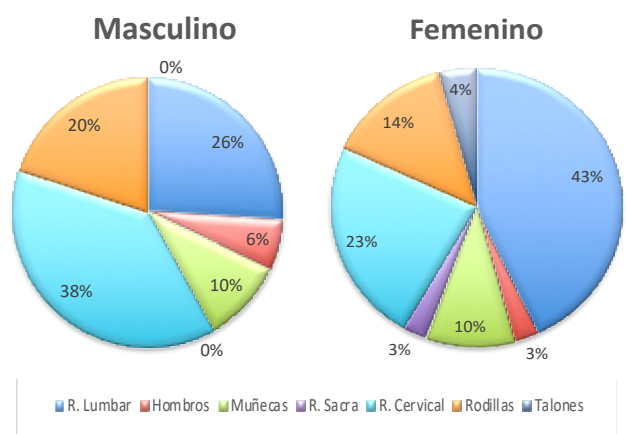

Fig. 4. Localización del dolor músculo-esquelético según el género

\section{Discusión}

El cuestionario COPCORD es una herramienta de tamizaje que tiene como finalidad identificar a las personas con dolor o malestar (inflamación, rigidez) músculoesquelético, candidatos para futuros estudios de confirmación de enfermedades reumáticas, su validez y confianza ha sido probada en varios países de África y Europa y se han realizado adecuaciones transculturales para su aplicación en varios países de Latinoamérica (México, Guatemala, Colombia, Brasil, Argentina, Chile, Perú y Ecuador), demostrando ser una prueba sensible y específica para la detección del dolor músculo-esquelético relacionado con patologías reumáticas. En nuestro país, específicamente en la ciudad de Cuenca, Guevara et al., ${ }^{6}$ reporta una sensibilidad para las enfermedades reumáticas del $83.7 \%$ y especificidad del $61.14 \%$ con una razón de verosimilitud de $2.18 \%$ y área bajo la curva de $0.72(0.66-0.78)$.

En el presente estudio, con la aplicación del cuestionario COPCORD se registra una prevalencia de DME del $61.93 \%$ cifras similares a los registrados por Londoño et al. ${ }^{11}$ en varias ciudades colombianas (prevalencia del $74 \%$ ), sin embargo, se le considera alta si se compara con otros estudios realizados en el continente como el de Granados et al., ${ }^{19}$ en una comunidad urbana de Venezuela (prevalencia del $22.4 \%$ ), Goicochea et al., ${ }^{9}$ en una población urbana de México (24.8\%), Peláez et al., ${ }^{17}$ en indígenas latinoamericanos (prevalencia del 28.2\%), Peláez et al., ${ }^{16}$ en 
ciudades Colombianas ( $43.6 \%$ ) con prevalencias menores a las encontradas en este estudio, incluso el estudio realizado por Guevara et al, ${ }^{15}$ en la ciudad de Cuenca (prevalencia del $32.5 \%$ ) reporta cifras más bajas, esta diferencia podría deberse al tipo de población estudiada, diferente etnicidad (blancos, mestizos, indígenas), el lugar de residencia (urbana, rural), el estatus socioeconómico, tipo de actividad laboral realizada y otras condiciones ambientales y genéticas que inciden directamente en la presencia de algunas patologías como la osteoartritis, la artritis reumatoide y la gota ${ }^{3}$ además en este estudio se evidencia una prevalencia alta de otras comorbilidades que también cursan con sintomatología osteomuscular, ${ }^{12,18}$ como son la diabetes, enfermedad renal crónica, enfermedades neurológicas y cáncer que pueden justificar estos resultados.

De acuerdo a las características demográficas, al igual que en otros estudios ${ }^{3,18,20}$ el DME predomina en el sexo femenino, sin embargo las diferencias no fueron estadísticamente significativas; se incrementa con la edad, siendo este el principal factor de riesgo identificado en este estudio, hallazgos similares se reportan en otras investigaciones $^{3,12,21}$ y revisiones sistemáticas testifican que a mayor edad, mayor es la prevalencia de DME reportando que entre el 25 y $80 \%$ de los ancianos presentarán en algún momento de su vida algún tipo de dolor músculoesquelético, condición en ocasiones subestimada por ser erróneamente considerada como parte del proceso natural del envejecimiento. ${ }^{22}$

La mayor parte de los encuestados realiza actividades laborales con intensidad moderada; como se evidencia, el DME está presenta en todos los grupos, siendo más frecuente en quienes realizan trabajos de intensidad leve en mujeres y de intensidad moderada en los varones, probablemente se debe al trabajo repetitivo, el estrés mecánico sostenido sobre articulaciones específicas y postura corporal inapropiada, factores que han sido asociados a ciertas afecciones como la osteoartritis ${ }^{18,21}$ principalmente de la rodilla $^{23}$ y otros problemas osteomusculares sobre todo lumbares y cervicales, reportándose en otras revisiones como la población más vulnerable la femenina, los funcionarios y trabajadores de oficina. ${ }^{1}$ Otras variables, como el lugar de trabajo no muestran una asociación estadísticamente significativa, presentando similar porcentaje tanto en el área rural como urbano (61 y $62 \%$ respectivamente), otros estudios reportan una mayor prevalencia de DME en el área urbana, ${ }^{21}$ la diferencia puede explicarse por el hecho de que en este estudio se consideró el territorio de trabajo en lugar de la residencia ya que la población pertenece al área urbana.

En cuanto a la localización del dolor, varía de acuerdo al género, en este estudio la principal región anatómica afectada fue la columna vertebral, específicamente la región cervical y lumbar, seguido de las rodillas, sin encontrar relación estadísticamente significativa con el género, intensidad laboral, ni con el lugar de trabajo; en otros estudios las rodillas representan la principal localización de dolor y están relacionados con las actividades tanto laborales como domésticas, 7,11,17,19,23 otros lugares frecuentes fueron hombros y manos.

La carga económica del DME está dada por su asociación a limitación funcional y discapacidad, observándose en este estudio que el $40.9 \%$ de individuos en algún momento presentó limitación funcional para ciertas actividades, hasta el $59 \%$ tuvo que ausentarse de su trabajo a causa del dolor y el $3.28 \%$ tiene discapacidad; en otros estudios la frecuencia de limitación funcional medida con HAQ-DI score (Health Assessment Questionnaire Disability Index) varia desde el $18.3 \%{ }^{16}$ hasta el $88 \%,{ }^{9}$ se relaciona con la localización del dolor y el tipo de enfermedad reumática, como lo reportado por Guevara et al., ${ }^{15}$ en la ciudad de Cuenca donde se detectó discapacidad física en el 6,1\% de la población estudiada y en el $63 \%$ de los pacientes con enfermedad reumática confirmada siendo más frecuente en la osteoartritis de la rodilla $(31.4 \%)$; Branco et al., ${ }^{8}$ en su estudio realizado en Portugal asevera que las patologías con mayor limitación funcional y discapacidad son la polimialgia reumática, la artritis reumatoide y la osteartrosis de la rodillas, su repercusión en la actividad laboral se determinó con el cálculo del promedio de número de días de absentismo laboral en los últimos 12 meses siendo de $31.5 \pm 83.9$ días (P: 0.141); mientras que Mathew et al., ${ }^{7}$ reveló que el $80 \%$ de los encuestados con DME exhibió algún grado de impacto en su capacidad para trabajar, el $8 \%$ tuvo que dejar de trabajar a causa del dolor con impacto económico en el $53 \%$ de casos. El incremento del gasto público con mayor consumo de recursos sanitarios también ha sido demostrado, Branco et al., ${ }^{8}$ reportó un mayor número de visitas médicas $(2.5 \pm 5.9)$, incremento de la frecuencia de hospitalizaciones $(11.4 \%)$ y necesidad de cuidados domiciliarios (2.7\%) en los últimos 12 meses; como se evidencia el DME al provocar limitación funcional y discapacidad influye directamente en la condición socioeconómica del paciente y su familia y altera su la calidad de vida.

En este estudio previo a la aplicación de la encuesta, el $45.7 \%$ tuvo algún diagnóstico ya instituido, siendo los más frecuentes la osteoartritis y la osteoporosis, información que coincide con otros estudios que señalan como las patologías reumáticas más reportadas a la osteoartritis seguida de dolor lumbar y síndrome de dolor regional. ${ }^{6,9,11,16-18}$

El DME en este estudio tiene una prevalencia alta, encontrándose como único factor relacionado y estadísticamente significativo la edad, deduciendo que, a mayor edad, mayor es la presencia de DME; no se encontró relación estadísticamente significativa con las otras variables demográficas ni con la intensidad laboral, probablemente debido a su fisiopatogénia autoinmune.

\section{Conclusión}

La prevalencia de DME es alta en este grupo poblacional, especialmente en las mujeres y en los grupos de mayor edad, es una causa importante de limitación funcional y 
discapacidad con gran repercusión en el condición socioeconómica del paciente, su familia y la sociedad; el cuestionario COPCORD ha demostrado en múltiples estudios, ser sensible y específico para detectar el DME de causa reumatológica, por lo que se sugiere su aplicación como herramienta de tamizaje, que permitirá el reconocimiento 10 y derivación oportuna a un Especialista para el tratamiento temprano, disminuyendo de esta manera la carga por estas enfermedades.

\section{Fuente de Financiamiento}

Este estudio es autofinanciado.

\section{Conflicto de Intereses}

No existen conflictos personales, profesionales, financieroso de otro tipo.

\section{Consentimiento Informado}

Los autores cuentan con el consentimiento informado de los pacientes para la investigación, la publicación del caso 13 . y sus imágenes.

\section{Referencias Bibliográficas}

1. León González M, Fornés Vives J. Estrés psicológico y problemática musculoesquelética. Revisión sistemática. Enfermeria Global. 2015;14(2):276-300.

2. Villaseñor J, Escobar V, De Lanza P, Guizar I. Síndrome de dolor miofascial. Epidemiología, fisiopatología, diagnóstico y tratamiento. Revista de Especialidades MédicoQuirúrgicas. 2013;18(2):148-157.

3. Ingram M, Symmons DPM. The burden of musculoskeletal conditions. Medicine (United Kingdom). 2018;46(3):152-155.

4. Muñoz J, Alpizar D. Síndrome Miofascial. Medicina Legal de Costa Rica. 2016;33(1).

5. Vergara L. Síndrome de dolor miofascial. Revista Hospital Clínico Universidad de Chile. 2018;29(1):60-69.

6. Guevara S, Feicán A, Peláez-Ballestas I, Ochoa V, Vintimilla F, Vintimilla J. Validación del cuestionario COPCORD para detección de enfermedades reumáticas. Cuenca - Ecuador. Revista de la Facultad de Ciencias Médicas Universidad de cuenca. 2014;32(2):18-29.

7. Mathew A, Chopra A, Thekkemuriyil D, George E, Goyal V, Nair J. Impact of musculoskeletal pain on physical function and health-related quality of life in a rural community in south India: A WHO-ILAR-COPCORD-BJD India study. Clinical Rheumatology. 2011;30(11):14911497.

8. Branco JC, Rodrigues AM, Gouveia N, Eusébio M, Ramiro S, Machado PM, et al. Prevalence of rheumatic and musculoskeletal diseases and their impact on healthrelated quality of life, physical function and mental health in Portugal: Results from EpiReumaPt- a national health survey. RMD open. 2016;2(1).
9. Goycochea-Robles M, Sanin L, Moreno-Montoya J, Alvarez-Nemegyei J, Burgos-Vargas R. Validity of the COPCORD Core Questionnaire as a classification tool for rheumatic diseases. Journal of Rheumatology. 2011;86:31-35.

0. Darmawan J. Recommendations from the Community Oriented Program for Control of Rheumatic Disease for data collection for the measurement and monitoring of health in developing countries. Clinical Rheumatology. 2007;26(6):853-857.

11. Londoño J, Peláez Ballestas I, Cuervo F, Angarita I, Giraldo R. Prevalencia de la enfermedad reumática en Colombia, según estrategia COPCORD-Asociación Colombiana de Reumatología. Estudio de prevalencia de enfermedad reumática en población colombiana mayor de 18 años. Revista Colombiana de Reumatología. 2018;25(4):245256.

12. Blyth FM, Briggs AM, Schneider CH, Hoy DG, March LM. The global burden of musculoskeletal pain-where to from here? American Journal of Public Health. 2019;109(1):35-40.

3. Peláez-Ballestas I, Pons-Estel BA, Burgos-Vargas R. Epidemiology of rheumatic diseases in indigenous populations in Latin-Americans. Clinical Rheumatology. 2016;35:1-3.

14. Casal J, Vasquez M. Abordaje del dolor musculoesquelético en urgencias. Emergencias. 2012;24:59-65.

15. Guevara-Pacheco SV, Feican-Alvarado A, Delgado-Pauta J, Lliguisaca-Segarra A, Pelaez-Ballestas I. Prevalence of disability in patients with musculoskeletal pain and rheumatic diseases in a population from Cuenca, Ecuador. Journal of Clinical Rheumatology. 2017;23(6):324-329.

16. Peláez Ballestas I, Santos A, Angarita Iea. Adecuación y validación transcultural del cuestionario COPCORD: Programa Orientado a la Comunidad para el Control de las Enfermedades Reumáticas en Colombia. Revista Colombiana de Reumatología. 2019;26(2):88-96.

17. Peláez-Ballestas I, Granados Y, Silvestre A, AlvarezNemegyei J, Valls E, Quintana R, et al. Culturesensitive adaptation and validation of the CommunityOriented Program for the Control of Rheumatic Diseases methodology for rheumatic disease in Latin American indigenous populations. Rheumatology International. 2014;34(9):1299-1309.

18. Chopra A, Abdel-Nasser A. Epidemiology of rheumatic musculoskeletal disorders in the developing world. Best Practice and Research: Clinical Rheumatology. 2008;22(4):583-604.

19. Granados Y, Cedeño L, Rosillo C, Berbin S, Azocar M, Molina ME, et al. Prevalence of musculoskeletal disorders and rheumatic diseases in an urban community in Monagas State, Venezuela: a COPCORD study. Clinical Rheumatology. 2015;34(5):871-877.

20. dos Reis-Neto ET, Ferraz MB, Kowalski SC, Pinheiro GdRC, Sato EI. Prevalence of musculoskeletal symptoms in the five urban regions of Brazil-the Brazilian 
COPCORD study (BRAZCO). Clinical Rheumatology.

Recibido: 5 de noviembre de 2019 2016;35(5):1217-1223.

21. Alonso Monteiro Bezerra M, Hellwig N, Da Rocha CasAceptado: 16 de diciembre de 2019 telar Pinheiro G, Souza Lopes C. Prevalence of chronic musculoskeletal conditions and associated factors in Brazilian adults - National Health Survey. BMC Public Health. 2018;18(1):1-10.

22. Arteaga C, Santacruz J, Ramirez L. Evaluación del dolor musculoesquelético en el anciano. Acta Médica Colombiana. 2011;36(1):30-35.

23. Haq SA, Davatchi F. Osteoarthritis of the knees in the COPCORD world. International Journal of Rheumatic Diseases. 2011;14(2):122-129. 
